\title{
Myosin 1f-mediated Activation of Microglia Contributes to the Photoreceptor Degeneration in a Mouse Model of Retinal Detachment
}

\section{Yimin Wang}

Shanghai First People's Hospital: Shanghai Jiaotong University First People's Hospital

\section{Xiaohuan Zhao}

Shanghai First People's Hospital: Shanghai Jiaotong University First People's Hospital

\section{Min Gao}

Shanghai First People's Hospital: Shanghai Jiaotong University First People's Hospital

\section{Xiaoling Wan}

Shanghai First People's Hospital: Shanghai Jiaotong University First People's Hospital

\section{Yinong Guo}

Shanghai First People's Hospital: Shanghai Jiaotong University First People's Hospital

\section{Yingying Qu}

Shanghai First People's Hospital: Shanghai Jiaotong University First People's Hospital

\section{Yuhong Chen}

Shanghai First People's Hospital: Shanghai Jiaotong University First People's Hospital

\section{Tong Li}

Shanghai First People's Hospital: Shanghai Jiaotong University First People's Hospital Haiyun Liu

Shanghai First People's Hospital: Shanghai Jiaotong University First People's Hospital

\section{Mei Jiang}

Shanghai First People's Hospital: Shanghai Jiaotong University First People's Hospital

\section{Feng Wang}

Shanghai First People's Hospital: Shanghai Jiaotong University First People's Hospital

\section{Xiaodong Sun ( $\nabla$ xdsun@sjtu.edu.cn )}

Shanghai Key Laboratory of Ocular Fundus Diseases, Shanghai General Hospital, Shanghai Engineering Center of Visual Science and Photomedicine, Shanghai 200080, China https://orcid.org/0000-00015015-0945

\section{Research}

Keywords: retinal detachment, photoreceptor degeneration, cell death, microglia, myosin $1 \mathrm{f}$ 
Posted Date: October 1st, 2020

DOl: https://doi.org/10.21203/rs.3.rs-80015/v1

License: (c) (i) This work is licensed under a Creative Commons Attribution 4.0 International License. Read Full License

Version of Record: A version of this preprint was published at Cell Death \& Disease on October 1st, 2021. See the published version at https://doi.org/10.1038/s41419-021-03983-3. 
1 Myosin 1f-mediated Activation of Microglia Contributes to the

2 Photoreceptor Degeneration in a Mouse Model of Retinal

3 Detachment

5 Yimin Wang, ${ }^{1,2,3,4,{ }^{*}}$ Xiaohuan Zhao, ${ }^{1,2,3,4,{ }^{*}}$ Min Gao, ${ }^{1}$ Xiaoling Wan, ${ }^{1,2,3}$ Yinong

6 Guo, 1,2,3 Yingying Qu,1,5 Yuhong Chen,1,2,3 Tong Li, 1,2 Haiyun Liu,1,2 Mei

7 Jiang, 1,2,3 Feng Wang, 5,\# Xiaodong Sun 1,2,3,4,\#

8 1. Shanghai General Hospital , Shanghai Jiao Tong University School of $9 \quad$ Medicine

10 2. National Clinical Research Center for Eye Disease

$113 . \quad$ Shanghai Key Laboratory of Ocular Fundus Diseases

12 4. Shanghai Engineering Center for Visual Science and Photomedicine

13 5. Shanghai Institute of Immunology, Translational Medicine Center, 14 Shanghai General Hospital, State Key Laboratory of Oncogenes and Related Genes, Shanghai Jiao Tong University School of Medicine

17 \#Corresponding author:

18 Dr. Xiaodong Sun and Dr. Feng Wang should be considered equivalent 19 corresponding authors.

21 Xiaodong Sun, xdsun@sjtu.edu.cn

22 Department of Ophthalmology, Shanghai General Hospital, Shanghai Jiao

23 Tong University School of Medicine, 100 Hai Ning Road, Shanghai 200080,

24 P.R. China

25 Tel: +86-021-36126305; Fax: +86-021-63240825

Feng Wang, wangfeng16@sjtu.edu.cn 
28 Shanghai Institute of Immunology, Translational Medicine Center, Shanghai 29 General Hospital, State Key Laboratory of Oncogenes and Related Genes,

30 Shanghai Jiao Tong University School of Medicine.

31 Tel: +86-021-53594658

32

33 * Yimin Wang and Xiaohuan Zhao contributed equally to this study and share 34 the first authorship.

35 Financial Disclosures:

36 There are no financial conflicts of interest to disclose.

38 Funding: This study was supported by the National Natural Science

39 Foundation of China ( 81730026, 81771739 ) National Science and

40 Technology Major Project (2017YFA0105301, 2019ZX09301113 ,

41 SQ2018YFA090045-01), Science and Technology Commission of Shanghai

42 Municipality ( $17411953000,19495800700,18 J C 1414100)$, the Program

43 for Professor of Special Appointments (Eastern Scholar) at Shanghai

44 Institutions of Higher Learning, the Top Young Talent Program of Shanghai,

45 and the Innovative Research Team of High-level Local Universities in

46 Shanghai. 
47 Abstract

48 Background: Photoreceptor death and neurodegeneration is the leading cause

49 of irreversible vision loss. The inflammatory response of microglia plays an

50 important role in the process of neurodegeneration. In this study, we examined

51 the involvement of myosin $1 \mathrm{f}$ as a key regulator of immune cell activation via

52 the AKT and MAPK pathways in microglia.

53 Methods: We chose retinal detachment as the model of photoreceptor

54 degeneration. Immunofluorescence and Western Blot was performed to

55 confirm the expression and location of myosin $1 \mathrm{f}$ in detached retina. The RD

56 mouse model was induced in WT and myosin 1f-/- mice and confirmed by HE

57 and TUNEL staining. The expression of inflammatory cytokine and downstream

58 pathways was assessed via qPCR and WB.

59 Results: Myosin 1f was upregulated after retinal detachment, and it was

60 specifically expressed in microglia. Deficiency of myosin $1 \mathrm{f}$ protected against

61 cell death by inhibiting microglia activation. The elimination of microglia can

62 abolish the protective effect of myosin $1 \mathrm{f}$ deficiency. After stimulation by LPS, 
63 microglia with myosin $1 \mathrm{f}$ deficiency showed downregulation of the MAPK and

64 AKT pathways.

65 Conclusions: Myosin $1 \mathrm{f}$ plays a crucial role in microglia-induced neuro-

66 inflammation after retinal injury and photoreceptor degeneration by regulating

672 classic pathways, MAPK and AKT, and thereby decreasing the expression of

68 inflammatory cytokines. Myosin $1 \mathrm{f}$ can be inhibited to prevent a decline in visual

69 acuity after photoreceptor degeneration.

70 Key Words: retinal detachment, photoreceptor degeneration. cell death,

71 microglia, myosin $1 f$ 


\section{Background}

The loss of photoreceptors and retinal function disorder is the feature of

74 photoreceptor degeneration, which leads to the irreversible vision loss [1]. The

75 pathogenesis is involved in many retinal diseases, including retinal detachment,

76 retinitis pigmentosa and age-related macular degeneration. In particular, retinal

77 detachment is a kind of disease that photoreceptors lose nutritional support

78 after being separated from the retinal pigment epithelium (RPE) layer and

79 choroidal vessels, which leads to the death of photoreceptors [2].

80 Rhegmatogenous retinal detachment (RRD) is the most common form of retinal

81 detachment (RD), with an incidence of 13 per 100,000 persons annually [3].

82 Although surgery can reattach the retina with a high success rate, a small

83 portion of patients still experience vision loss due to photoreceptor death $[4,5]$.

84 Therefore, discovering the mechanisms of the process of cell death is crucial

85 to neuroprotection and intervention.

86 Although many death effectors have been discovered and targeted to

87 prevent the loss of photoreceptors, little progress on rescuing photoreceptor 
88 function after retinal injury [6]. Recently, transcriptome analysis has revealed

89 that inflammatory responses play an important role in the process of

90 photoreceptor degeneration [1, 7]. Many chemokines - such as TNF- $\alpha$, IL-1 $\beta$,

91 IL-6, IL-8, and MCP-1 — can reach a high level after 1 hour following retinal

92 detachment [8, 9]. These cytokines can activate microglia and recruit

93 macrophages in the sub-retinal space [1]. In the meantime, the activated

94 microglia can release MCP-1, which contributes to the increased expression of

95 MCP-1 in Müller cells and macrophages [10]. The positive feedback aggravates

96 immune response and maintains a high level of neuro-inflammation [1, 11],

97 which can be harmful to photoreceptors [2].

98 Microglia play a key role in the inflammatory feedback loop, which is a

99 potential therapeutic target for neuroprotection in retinal degeneration.

100 Activated microglia can express TNF- $\alpha$ and IL-1 $\beta$, which are widely

101 implicated in retinal degenerative disease. TNF- $\alpha$ can combine with TNF

102 receptors 1 and 2, which are distributed on the membranes of neurons, leading

103 to cytotoxicity by causing mitochondrial dysfunction and oxidative stress. IL-1 $\beta$ 
104 is able to combine with interleukin receptors and leads to cell death in a

105 manner similar to TNF- $\alpha$ signaling $[12,13]$. Meanwhile, it secretes MCP-1 to

106 recruit and activates astrocytes and immune cells from circulation, aggravating

107 the immune response $[1,11,14]$. Thus, it is important to discover the

108 mechanisms by which microglia affect this loop in order to potentially inhibit the

109 over-activation of microglia and the release of toxic cytokines. Mitogen-

110 activated protein kinases (MAPKs) regulates the expression of pro-

111 inflammatory cytokines such as TNF- $\alpha$, IL-1 , and IL-6 [15]. Activated protein

112 kinase $B(P K B)$, also named as $A K T$, can regulate inflammatory response by

113 downstream factors[16]. However, the upstream mechanism is not entirely

114 understood.

115 The myosin family, as a component of the cytoskeleton, has been reported

116 to play a crucial role in cell signaling $[17,18]$. It is also a well-known component

117 of skeletal muscle in particular, in which it facilitates contractions [19]. In

118 addition, the delivery of intercellular material cannot occur without myosins;

119 they help the vesicles transport materials along the actin filament tracks [20]. 
120 Class 1 myosins have been revealed to be key components during pinocytosis

121 [21], phagocytosis [22], cell motility [23], and secretion [24]. Therefore, the

122 exploration of myosin function in microglia can lead to a better understanding

123 of pathogenesis after retinal detachment. In this study, we have proven that

124 myosin $1 \mathrm{f}$ is involved in the activation of microglia by regulating the MAPK and

125 AKT pathways in mouse models of photoreceptor degeneration.

126

127 Methods

128 Sequencing data extraction and bioinformatics analysis

129 The sequencing data (GSE28133) were downloaded from GEO

130 (https://www.ncbi.nlm.nih.gov/geo/query/acc.cgi?acc=GSE28133), which is a

131 public database of chips and microarrays. The data contain 38 human retinal

132 samples, including 19 samples from RD patients and 19 control samples

133 without RD.

134 The data were normalized first, then DEGs were analyzed by a limma

135 algorithm using the $\mathrm{R}$ programming language. The log fold change cutoff and 
136 adjusted $p$ value were set as 1 and 0.05 , respectively. Points without gene

137 symbols were removed.

138 A GO enrichment analysis of DRGs was obtained using the online tool

139 DAVID (https://david.ncifcrf.gov /home.jsp, version 6.8). The bubble maps were

140 drawn using Hmisc and ggplot2 via the R programming language. The GSEA

141 analysis was conducted using GSEA_4.0.1 software.

142

143 Retinal detachment model animals

144 Myosin 1f-/- mice were purchased from Jackson Laboratory, then bred in

145 the Shanghai General Hospital animal facility. All animal experiment protocols

146 were in agreement with the Statement of the Association for Research in Vision

147 and Ophthalmology for biomedical research. The animals were randomly

148 assigned into 2 groups.

149 We used 1\% atropine sulphate oculentum (Santen, Japan) and 0.5\%

150 tropicamide (Santen, Japan) on the ocular surface of mice to dilate the pupil.

151 We applied $0.4 \%$ oxybuprocaine eye drops (Santen, Japan) as a surface 
152 anesthesia. The mice were anesthetized using isoflurane gas (1.5\% mixed with

$15350 \%$ air and $50 \% \mathrm{O}^{2}$ ). We applied $0.3 \%$ ofloxacin oculentum (Shanghai, China)

154 as a magnifying lens to obtain a clearer view.

155 The examination of the RD model was conducted as previously described

$156[25,26]$. The sclera was exposed and punctured at $2 \mathrm{~mm}$ posterior to the limbus

157 with a 34-gauge needle. The vitreous humor was slowly aspirated with a 34G

158 glass needle until the retina separated spontaneously from the underlying RPE

159 layer. Then the $34 \mathrm{G}$ needle tip was inserted into the subretinal space through

160 the same scleral hole, and sodium hyaluronate (HA, Shanghai, China)

161 was gently injected. The fundus was observed and injection was halted after

162 the retina was detached in every quadrant. The scleral hole was then sealed

163 using cyanoacrylate surgical glue to prevent HA leakage. Finally, tobramycin

164 and dexamethasone ointments (Alcon, USA) were applied to the ocular surface

165 to prevent infection.

166

167 Immunofluorescence 
$16910 \mu \mathrm{m}$ to fabricate eye sections. We removed the chamber and kept the entire

170 retina to prepare for the retinal stretched preparation after fixation.

171 We stained for the following antibodies: IBA-1 (1:1,000, Wako, 019-19741),

172 F4/80 (1: 1,000, Abcam, 6640), GFAP (1: 1,000, Abcam, 4674), and myosin 1f

173 (1: 1,000, Abcam, ab197215). The immunofluorescence was observed under a

174 confocal microscope (Leica TCS SP8 confocal 137 microscope, Germany) and

175 quantitatively analyzed using ImageJ software (Fiji, NIH, USA).

176

177 HE stains and ONL thickness

178 After being fixed in 4\% paraformaldehyde, the eyeballs were embedded in

179 paraffin and sectioned into $10-\mu \mathrm{m}$ slices. The eye sections were stained with

180 hematoxylin and eosin. We measured 10 points of thickness within the outer

181 nuclear layer (ONL) on 1 section with the same spacing distance. The

182 measurements were taken using ImageJ software (Fiji, NIH, USA). 

sections to detect apoptosis. The sections were permeated with $0.1 \%$ Triton $\mathrm{X}$ 100 in $1 \%$ sodium citrate for 10 minutes, then they were incubated with a

188 TUNEL reaction mixture for 1 hour at $37^{\circ} \mathrm{C}$. The sections were observed under

189 the confocal microscope, and all TUNEL-positive cells were counted.

193 because BV2 can be a good substitute for primary microglia in many

194 experimental settings [27]. Small interfering RNA (siRNA) of myosin 1f (768:

195 CCACAUCUACUACCAGCUUTT AAGCUGGUAGUAGAUGUGGTT; 1413:

196 GCAGGAGGAGUAUGUGCAATT UUGCACAUACUCCUCCUGCTT; 2662:

197 GCGGACAGCUUCUUAGAAATT UUUCUAAGAAGCUGUCCGCTT) and

198 TransIT-2X (MIR 6000, Mirus) were chosen for the knockdown of myosin $1 \mathrm{f}$. 
199 For stimulation, $100 \mathrm{ng} / \mathrm{ml}$ of lipopolysaccharide (LPS, L2880, Sigma Aldrich,

200 St. Louis, USA) was applied after 24 hours.

202 Western blot analysis

203 Retina and cell samples were lysed in a lysis buffer containing $50 \mathrm{mM}$ Tris-

$204 \mathrm{HCl}(\mathrm{pH} 8.0)$ and $0.1 \%$ SDS, as well as the complete Protease Inhibitor Cocktail

205 (11697498001; Roche Applied Science), $150 \mathrm{mM} \mathrm{NaCl}, 1 \%$ Triton X-100, and

$2061 \%$ sodium deoxycholate. We incubated the primary antibodies overnight. The

207 antibodies were as follows: GAPDH (Proteintech, 60004-1-lg, RRID:

208 AB_2107436; Proteintech, Chicago, IL, USA), AKT (C67E7, Rabbit mAb, CST),

209 p-AKT (Ser473, D9E, XP, Rabbit mAb, CST), ERK1/2 (137F5, Rabbit mAb,

210 CST), p-ERK1/2 (D13.14.4E, XP, Rabbit mAb), JNK (\#9252, CST), p-JNK

211 (81E11, Rabbit mAb \#4668, CST). 
214 TNF- $\alpha$ and IL-1 $\beta$ were detected by using ELISA kits according to the

215 manufacturer's protocols (MTA00B, MLB00C, R\&D Systems, Minneapolis,

216 USA). The tissue samples were lysed in PBS, and the supernatant was

217 collected. The cell samples were then collected from the cultural supernatant.

219 Quantitative real-time PCR

220 The primer sequences were acquired from Primerbank

221 (bankhttps://pga.mgh.harvard.edu/primerbank/), including TNF- $\alpha$ (F:

222 CCCTCACACTCAGATCATCTTCT, R: GCTACGACGTGGGCTACAG), IL-1 $\beta$

223 (F: GAAATGCCACCTTTTGACAGTG, R: TGGATGCTCTCATCAGGACAG),

224 IL-6 $\quad$ F: $\quad$ TAGTCCTTCCTACCCCAATTTCC, $\quad$ R:

225 TTGGTCCTTAGCCACTCCTTC), and myosin if (F:

226 CTTTCACTGGCAGAGTCACAA, R: ATGAAGCGTTTGCGGAGGTT). 
230 optical coherence tomography were performed on eyes with dilated pupils. The

231 equipments were purchased from Phoenix Research Labs, Inc. systems

232 (Phoenix, USA).

234 Data and statistical analysis

235 The statistical analysis was conducted using Prism8 software. The data are

236 presented as mean \pm SEM, unpaired t-test, ${ }^{*} p<0.05,{ }^{* *} p<0.01,{ }^{* *} p<0.001$,

$237{ }^{* * * *} p<0.0001 ; p>0.05$ was regarded as insignificant.

\section{Results}

240 Myosin $1 f$ is upregulated after retinal detachment

241 We analyzed the data of GSE28133 from GEO datasets, which included 19

242 retinal tissues from retinal detachment patients and 19 control samples without

243 ocular disease. A differential expression analysis revealed that 990 genes were

244 upregulated and 272 genes were downregulated ( $|\mathrm{FC}|>1.5$ and $p<0.05)$ (Figure 
245 1A). As we expected, GO analysis indicated that immune response was

246 involved in the pathology of RD (Figure 1B, 1C). GSEA enrichment analysis

247 also confirmed the results (Figure 1D) that immune response was positively

248 correlated to the RD group (Supplementary 1, Figure A). To discover the

249 expression pattern of myosins after RD, a heat map was examined and

250 revealed that several myosins were upregulated, including MYO1F, MYO3A,

251 and MYO5C, in the RD groups (Figure 1E), and MYO1F was the most

252 upregulated among the 3 , with an FC of 1.786 (Figure 1F). To figure out the

253 expression pattern of MYO1F along with time, we divided the patients into 3

254 groups according to the RD duration, including within one month, 1 month to 3

255 months and more than 3 months. The fold change MYO1F is the highest among

256 the patients with a RD duration within one month ( $F C=2.19493181)$ (Figure 1G),

257 suggesting an upregulation of MYO1F in the early phase.

258 To verify the results of the RNA sequencing, we detected the expression of

259 myosin $1 \mathrm{f}$ in mouse retina samples. A western blot revealed that myosin $1 \mathrm{f}$ was

260 upregulated on the detached retina, and it reached the peak at day 3 after 
261 detachment (Figure 2A). Retinal injury always occurs with inflammation

262 because microglia can be activated after the injury. We examined the location

263 of myosin 1f via immunofluorescence. Immunofluorescence confirmed that

264 myosin $1 f$ was upregulated within the retinal section after RD, and it was co-

265 located with microglia markers, including F4/80 and IBA1 (Figure 2B, C). Thus,

266 myosin $1 \mathrm{f}$ is specifically expressed in microglia; there was no sign of co-location

267 between myosin 1f and GFAP (Figure 2D).

268 We also confirmed the expression of myosin $1 \mathrm{f}$ in the other 2 models of

269 photoreceptor degeneration. $R d 1$ mouse is a model for retinitis pigmentosa,

270 which is also characterized by photoreceptor death and microglia activation [28].

271 Light damage is another model of photoreceptor degeneration [29, 30]. We

272 discovered the same expression pattern of myosin $1 \mathrm{f}$ in both the $r d 1$ mouse

273 model (Figure 2E) and the light damage model (Figure 2F). Similarly, myosin

2741 was expressed on F4/80 positive cells within the retina sections of the $r d 1$

275 mouse model (Figure $2 \mathrm{G}$ ) and the light-damaged eye (Figure $2 \mathrm{H}$ ). 
To further study the function of myosin $1 \mathrm{f}$, we investigated its effect on

279 photoreceptor death in a myosin $1 \mathrm{f}-/$ - mouse model [18, 31, 32]. To verify that

280 myosin $1 \mathrm{f}$ deficiency does not contribute to retinal injury and photoreceptor

281 death, we observed the structure and function of the retina. Twenty-week-old

282 myosin 1f-/- mice were compared with age-matched wild types and showed

283 normal structures on both optical coherence tomography (Supplementary 2,

284 Figure A, B) and HE section (Supplementary 2, Figure C, D), and the amplitude

285 of electroretinograms (ERGs) for both groups were within the normal range

286 (Supplementary 2, Figure E).

We chose RD model as a representative model of photoreceptor

288 degeneration, then observed the myosin 1f-/- mice at 3 days after RD (Figure

$2893 \mathrm{~A}$ ) because it is reported that the apoptosis of photoreceptors is the most

290 extensive at the 3rd day. Fundus photography and HE staining showed that the

291 retina remained detached at the 3rd day (Supplementary 3, Figure A, B). To

292 assess photoreceptor loss, we calculated the thickness of the outer nuclear 
293 layer (ONL) (Figure 3B). Myosin 1f-/- mice exhibited thicker ONLs after RD

294 (Figure 3C). In addition, there were fewer TUNEL-positive cells in the myosin

295 1f-/- (Figure 3D, 3E), which suggests that myosin $1 \mathrm{f}$ deficiency can protect

296 against photoreceptor apoptosis.

298 Myosin 1f influences microglia activation

299 We analyzed the morphology of microglia 3 days after RD via stretched

300 preparation and immunofluorescence. The microglia were more extended and

301 had more branches in the retina of myosin $1 \mathrm{f}-/-$ mice, whereas the microglia of

302 WT retina were more shrunken and tended to be round (Figure 4A). A

303 quantitative skeleton analysis and a Sholl analysis showed more intersections

304 in the myosin 1f-/- group (Figure 4B, 4C). This indicates that myosin 1f may

305 contribute to microglia activation. We have also counted the IBA1+ cells of the

306 stretched preparation and cell counts on the retina of myosin 1f-/- showed no

307 difference (Supplementary 3, Figure C). Microglia can migrate to injury site after 
$308 \mathrm{RD}$, especially outer nuclear layer (ONL), however, we have not observed the

309 difference in numbers of infiltrated microglia (Supplementary 3, Figure D).

310 To evaluate the inflammation status of the retina and the function of

311 microglia in the myosin 1f-/- group after RD, we detected the expression levels

312 of inflammation markers and signature cytokines. IL-1 $1 \beta$, IL- 6 and TNF- $\alpha$ are

313 classic pro-inflammatory cytokines in activated microglia [33]. The ELISA

314 analysis suggests IL-1 $\beta$ (Figure 4D) and TNF- $\alpha$ (Figure 4E) was also

315 downregulated in myosin 1f-/- mice at day 3 . The mRNA expression is in

316 accordance with the protein expression pattern. We observed the

317 downregulation of IL-1 $\beta$, TNF- $\alpha$, and IL-6 in myosin 1f-/- mice at day 3 ,

318 compared to WT mice (Figure 4F). In addition, CD68, a marker related to the

319 degree of activation [34, 35], was lower at day 1 in myosin 1f-/- mice (Figure 320 4G). 
To verify that myosin $1 \mathrm{f}$ deficiency can protect photoreceptors by regulating

324 the activation of microglia, we administered PLX3397 [36] to mice via oral

325 gavage to eliminate mononuclear phagocytes, including microglia. PLX3397 is

326 an inhibitor of the CSF1R receptor, which is widely expressed in mononuclear

327 phagocytes [37]. It is reported that PLX3397 can reduce tissue macrophages

328 without affecting myeloid cells [38]. We started gavage at day 1 , and a dose

329 was delivered every day until day 7 . We conducted the RD model experiment

330 at day 4 and observed at day 7 (Figure $5 \mathrm{~A}$ ).

331 The retinal thickness and the TUNEL-positive cells were calculated to

332 evaluate photoreceptor death. There was no difference in ONL thickness

333 between the 2 groups after PLX3397. Likewise, there were no significant

334 differences in TUNEL-positive cell counts between the 2 groups (Figure 5B,

335 5C). We then detected mRNA expression after microglia elimination.

336 Interestingly, the difference in CD68 between the 2 groups disappeared (Figure

337 5D). Similarly, the mRNA expression of IL-1 $\beta$ and TNF- $\alpha$ also disappeared 
338 (Figure 5E). That is to say, microglia elimination abolished the protective effect

339 of myosin $1 \mathrm{f}$ deficiency.

341 Myosin 1 affects microglia activation by regulating the MAPKV AKT pathways

342 To further explore the molecular mechanism underlying myosin $1 \mathrm{f}$ -

343 mediated photoreceptor degeneration, we used lipopolysaccharide (LPS), a

344 pathogen-associated molecular pattern (PAMP) to stimulate BV2 cell lines in

345 vitro. BV2 is an immortalized cell line derived from mice [39]. We noticed the

346 upregulation of myosin $1 \mathrm{f}$ after stimulation (Figure 6A). The transcription level

347 of IL-1 $\beta$ rose 6 hours after stimulation. In addition, an ELISA analysis suggests

348 that TNF- $\alpha$ rose after LPS stimulation (Figure 6B). Furthermore, we developed

349 a siRNA knockdown system for myosin 1f. We designed 3 sequences of siRNA,

350 including 768,1413 , and 2662 ; all of them led to a significant decrease of

351 myosin $1 f$ at the protein level (Figure 6C). We chose 2662 to detect the

352 quantitative efficiency of the siRNA, and the efficiency reached about $80 \%$

353 (Figure 6D). 
354 We used LPS to stimulate the control and myosin $1 \mathrm{f}$ knockdown cells. Then

355 we detected the expression of IL-1 $\beta$ and TNF- $\alpha$ in the cellular supernatant at

356 hour 24 via ELISA, and we found that both cytokines went down in the

357 knockdown group (Figure 6E).

358 Previous studies showed that MAPK and AKT signaling pathways have

359 been implicated in the activation of microglia. We then detected the expression

360 of proteins related to MAPK and AKT. The proportion of phospho-AKT

361 decreases after 24 hours of LPS stimulation (Figure 6F). As the 2 main

362 components of MAPK, phospho-ERK and phospho-JNK also showed

363 significant decreases. In addition, we repeated western blot to detect the

364 phosphorylation protein in the detached retina of WT and myosin if mice

365 (Figure 6G). In vivo results may further verify the consequences of myosin $1 \mathrm{f}$

366 regulation of microglia activity through the MAPK and AKT pathways. 
370 upregulated after photoreceptor degeneration in both human retina and mouse

371 model. We further demonstrated that myosin $1 \mathrm{f}$ can regulate microglia

372 activation, whereas the absence of myosin $1 f$ can protect photoreceptors by

373 inhibiting the MAPK and AKT pathways and decreasing the expression of

374 inflammatory cytokines, such as TNF- $\alpha$ and IL-1 $\beta$, in microglia.

375 The vision loss after RD is mainly due to photoreceptor death [40]. Various

376 forms of cell death are involved in this pathology, including apoptosis, necrosis,

377 and autophagy, which peak at 2 to 3 days after RD [41]. Despite the discovery

378 and inhibition of downstream effectors in cell death, no current techniques can

379 stop photoreceptor death completely [6]. This indicates that cell death is a 380 complicated process involving different pathologies, including inflammation.

381 The sequence of the human retina sample after RD demonstrated that immune

382 response and neurodegeneration are 2 major biological processes involved in 383 RD [7]. 
384 Besides, we have discovered that myosin $1 f$ was up-regulated after

385 photoreceptor degeneration. Myosins, as a family of molecular motors, have

386 various functions in the cell, including cell transformation, cell motility, material

387 transportation within cells, and signal transduction [42]. Furthermore, myosin 1f,

388 a class 1 myosin, can regulate the immune response $[43,44]$. It is reported that

389 myosin $1 \mathrm{f}$ is not only involved in the M1 polarization of macrophages by

390 stimulating intercellular adhesion [45] but also influences neutrophil migration

391 [32]. In our study, we found that myosin $1 f$ was the most upregulated myosin

392 by re-analyzing human RD sequence data, and we verified the results in a

393 mouse RD model. We also found that myosin $1 \mathrm{f}$ is generally upregulated in $r d 1$

394 mutation mouse model and light-induced retinal injury. The rd1 mutation mouse

395 is a classical model for retinitis pigmentosa, with the rod photoreceptor

396 degeneration at postnatal day 8[46]. Microglia are activated and express

397 inflammatory cytokines in rd1 mouse model retina. A similar pathology occurs

398 in light-induced injury. Thus, we discovered that myosin $1 f$ was also

399 upregulated in the 2 latter cases, which suggests it could play a key role in 
400 photoreceptor degeneration. It is reported that myosin $1 \mathrm{f}$ is also upregulated in

401 brain neurodegeneration, such as Alzheimer's disease (AD), Huntington's

402 disease (HD), and Parkinson's disease (PD) [47], suggesting the upregulation

403 of myosin $1 \mathrm{f}$ is a common phenomenon in neuro-inflammation.

404 We also discovered that myosin $1 \mathrm{f}$ is highly expressed in microglia. Via

405 immunofluorescence, we found that myosin $1 \mathrm{f}$ displayed a strong co-

406 localization with mononuclear macrophages but not Müller cells, indicating a

407 possible relationship between myosin $1 \mathrm{f}$ and microglia activation after retinal

408 injury. Though myosin $1 \mathrm{f}$ is involved in the motility of neutrophils[31], it is

409 surprising that myosin $1 \mathrm{f}$ does not affect the migration of microglia to injury site.

410 Instead, it affects the morphology of microglia, suggesting that the function of

411 myosin $1 \mathrm{f}$ could be various among different immune cell types.

412 To further determine the function of myosin $1 \mathrm{f}$, we used a myosin $1 \mathrm{f} \mathrm{KO}$

413 mouse model to observe the difference. The KO mouse model showed a

414 significant reduction in neuron death. It suggested that myosin $1 \mathrm{f}$ deficiency 
415 could be protective to neurons and it is possible to work by affecting the

416 activation of microglia.

417 Microglia, as a resident immune cell, is among the main effector cells of

418 neuroinflammation after retinal injury [48]. In our study, we have discovered that

419 myosin 1f deficiency have reduced the expression of inflammatory cytokines

420 such as TNF- $\alpha$ and IL-1 $\beta$. Besides, we found the morphological difference of

421 microglia in myosin $1 \mathrm{f} \mathrm{KO}$ retina and WT retina after RD. After the elimination

422 of microglia, the protective effect of myosin $1 \mathrm{f} \mathrm{KO}$ disappeared. Therefore, we

423 think myosin $1 \mathrm{f}$ can regulate the activation of microglia and involved in neuro-

424 inflammation, which leads to the death of photoreceptors. In fact, the function

425 of microglia in physiological and pathological conditions remains unclear [49].

426 It is a double-edged sword; although over-activated microglia can release

427 cytotoxic factors that lead to neuron death [50,51], it can also protect neurons

428 by secreting neuroprotective factors and phagocytosing injured cells [52]. The

429 state of microglia can be decided by the course of the disease, the immune

430 microenvironment, and interactions with other cells. Still and all, the two states 
431 of microglia are dynamic and coexist. Discovering a way to inhibit the

432 inflammation pathway is still a promising target for retinal degeneration.

433 We discovered that myosin $1 \mathrm{f}$ can regulate the MAPK and AKT pathway to

434 promote the transcription of pro-inflammatory cytokines and activate microglia.

435 Mitogen-activated protein kinases (MAPK) signal the transduction pathway,

436 which consists of ERKs, c-Jun NH2-terminal kinases (JNKs), and p38 MAPKs

437 and is reported to be related to inflammation, cell proliferation, and apoptosis

$438[53,54]$. It is also associated with microglia-induced neuro-inflammation and

439 the secretion of neurotoxic cytokines $[55,56]$. AKT is an important protein of

440 signaling transduction, which is involved in multiple pathways[16], and it can

441 promote the expression of pro-inflammatory cytokines [57]. AKT can also be an

442 upstream regulator of NF- $\kappa b$ pathway [58]. NF- $\kappa b$ is a classic, crucial

443 transcription factor in both innate and adaptive immune responses, and it

444 participates in microglia induced neuro-inflammation [59, 60]. Our results reveal

445 that myosin $1 f$ could be the common upstream of MAPK and AKT pathways,

446 making it a promising target for neuroprotective approaches. 
Myosins can influence signaling in several ways, through phosphorylation,

448 receptor recycling, interaction with integrin, and so on [61, 62]. Class II myosin

449 can increase the integrin $\beta 1$ activity by clustering, and integrin $\beta 1$ is required

450 for the activation of AKT pathway[62]. Myosin 1C, myosin 1E and myosin $1 \mathrm{G}$

451 can regulate TGF- $\beta$ signaling by regulating the recycling and redistribution of

452 TGF- $\beta$ receptors to cell membrane. Myosin $1 f$ may work in a similar way,

453 however, more future researches are needed to explore the interaction of

454 myosin $1 f$ and downstream pathways.

455 In this study, we have revealed the the function of myosin $1 \mathrm{f}$ in neuro-

456 inflammation, through the regulation of microglia activation. We wondered that

457 the up-regulation of myosin $1 \mathrm{f}$ may be universal in other retinal degeneration

458 models, such as light damage and $r d 1$ mouse model, since microglia is usually

459 activated when encountering retinal injury. Our finding may provide a new

460 perspective for neuro-protection in photoreceptor degeneration. It is reported

461 that several compounds have been discovered as the inhibitors of myosins,

462 such as pentachloropseudilin (PCLP), a pseudilin derivative, which is a class 
463 one myosin specific inhibitor [63, 64]; Blebbistatin [65], a myosin-2 inhibitor;

464 Azidoblebbistatin, a photoreactive myosin inhibitor [44]. Our data suggest that 465 myosin $1 \mathrm{f}$ may be a novel pharmacological target for protecting photoreceptors 466 and preserving visual acuity.

\section{Conclusions}

In summary, our results show that myosin $1 \mathrm{f}$ plays a crucial role in microglia-

470 induced neuro-inflammation after retinal injury and photoreceptor degeneration

471 by regulating 2 classic pathways, MAPK and AKT, and thereby decreasing the

472 expression of inflammatory cytokines. Knockout of myosin $1 \mathrm{f}$ reduces the

473 intensity of the immune response and prevents cell death, suggesting that

474 myosin $1 f$ can be inhibited to prevent a decline in visual acuity after 475 photoreceptor degeneration. 
479

$480 \quad$ Funding

481 This study was supported by the National Natural Science Foundation of China

482 ( 81730026 , 81771739 ) National Science and Technology Major Project

483 (2017YFA0105301 , 2019ZX09301113 , SQ2018YFA090045-01),Science and

484 Technology Commission of Shanghai Municipality ( 17411953000 , 19495800700,

485 18JC1414100), the Program for Professor of Special Appointments (Eastern Scholar) at

486 Shanghai Institutions of Higher Learning, the Top Young Talent Program of Shanghai, and

487 the Innovative Research Team of High-level Local Universities in Shanghai.

488

489 Abbreviations

490 AD: Alzheimer's disease

491 HD: Huntington's disease

492 JNK: c-Jun NH2-terminal kinase

493 LPS: lipopolysaccharide

494 MAPK: mitogen-activated protein kinase 
495 ONL: outer nuclear layer

496 PAMP: pathogen-associated molecular pattern

497 PCLP: pentachloropseudilin

498 PD: Parkinson's disease

499 PKB: protein kinase $B$

500 RPE: retinal pigment epithelium

501 RD: retinal detachment

502 RRD: rhegmatogenous retinal detachment

504 Availability of Data and Materials

505 The datasets analyzed during the current study are available from the corresponding

506 author on reasonable request.

508 Ethics approval and consent to participate

509 All animal experiments were approved by the Ethics Committee of Jiao Tong University,

510 Shanghai, China, and were conducted in compliance with the Association for Research in 
511 Vision and Ophthalmology Statement for the Use of Animals in Ophthalmic and Vision

512 Research.

513

$514 \quad$ Competing interests

515 The authors declare that they have no competing interests.

516

517 Consent for publication

518 Not applicable.

519

520 Authors' contributions

521 All authors read and approved the final manuscript.

522 Yimin Wang and Xiaohuan Zhao had full access to all of the data and take responsibility

523 for the integrity of the data and the accuracy of the data analysis.

524 Concept and design: Yimin Wang, Xiaohuan Zhao, Feng Wang, Xiaodong Sun.

525 Acquisition, analysis, or interpretation of data: Yimin Wang, Xiaohuan Zhao, Min Gao,

526 Yinong Guo, Yingying Qu, Yuhong Chen, Tong Li, Haiyun Liu. 
Drafting of the manuscript: Yimin Wang.

528 Critical revision of the manuscript for important intellectual content: Xiaoling Wan, Mei

529 Jiang, Feng Wang, Xiaodong Sun.

530 Statistical analysis: Yimin Wang, Xiaohuan Zhao

531 Obtained funding: Xiaodong Sun

532 Dr. Xiaodong Sun and Dr. Feng Wang are corresponding authors.

533

534 Authors' information

535 Yimin Wang, Xiaohuan Zhao, Min Gao, Xiaoling Wan, Yinong Guo, Yingying Qu, Yuhong

536 Chen, Tong Li, Haiyun Liu, Mei Jiang, Feng Wang, Xiaodong Sun 


\section{Reference}

538

539

540

541

542

543

544

545

546

547

548

549

550

551

552

553

554

555

556

557

558

559

560

561

562

563

564

565

566

1. Sene, A. and R. S. Apte, Inflammation-Induced Photoreceptor Cell Death. Advances in experimental medicine and biology, 2018. 1074: p. 203-208.

2. Murakami, Y., et al., Photoreceptor cell death and rescue in retinal detachment and degenerations. Progress in Retinal and Eye Research, 2013. 37: p. 114-140.

3. Vail, D., et al., The Relative Impact of Patient, Physician, and Geographic Factors on Variation in Primary Rhegmatogenous Retinal Detachment Management. Ophthalmology, 2020. 127 (1): p. 97-106.

4. Soni, C., D. P. Hainsworth, and A. Almony, Surgical management of rhegmatogenous retinal detachment: a meta-analysis of randomized controlled trials. Ophthalmology, 2013. 120(7): p. 1440-1447.

5. Geiger, M., et al., Predictors for recovery of macular function after surgery for primary macula-off rhegmatogenous retinal detachment. International Ophthalmology, 2019. 40(3): p. 609616.

6. Wubben, T. J., C. G. Besirli, and D. N. Zacks, Pharmacotherapies for Retinal Detachment. Ophthalmology, 2016. 123(7): p. 15531562.

7. Delyfer, M. -N., et a1., Transcriptomic analysis of human retinal detachment reveals both inflammatory response and photoreceptor death. P1oS one, 2011. 6(12): p. e28791.

8. Yoshimura, T., et al., Comprehensive analysis of inflammatory immune mediators in vitreoretinal diseases. PloS one, 2009. 4(12) : p. e8158.

9. Nakazawa, T., et al., Attenuated glial reactions and photoreceptor degeneration after retinal detachment in mice deficient in glial fibrillary acidic protein and vimentin. 
Investigative ophthalmology \& visual science, 2007. 48(6): p.

570

571

572

573

574

575

576

577

578

579

580

581

582

583

584

585

586

587

588

589

590

591

592

593

594

595

596

597

598

599

600

601

2760-2768.

10. Wang, M., et al., Adaptive Müller cell responses to microglial activation mediate neuroprotection and coordinate inflammation in the retina. Journal of neuroinflammation, 2011. 8: p. 173.

11. Rutar, M., R. Natoli, and J.M. Provis, Small interfering RNAmediated suppression of Ccl2 in Müller cells attenuates microglial recruitment and photoreceptor death following retinal degeneration. Journal of neuroinflammation, 2012. 9: p. 221.

12. Rathnasamy, G., et al., Retinal microglia - A key player in healthy and diseased retina. Progress in neurobiology, 2019. 173: p. $18-40$.

13. Karlstetter, M., et al., Retinal microglia: just bystander or target for therapy? Progress in retinal and eye research, 2015. 45: p. $30-57$.

14. Vecino, E., et al., Glia-neuron interactions in the mammalian retina. Progress in retinal and eye research, 2016. 51.

15. Lai, J.-L., et a1., Indirubin Inhibits LPS-Induced Inflammation via TLRA Abrogation Mediated by the NF- $k B$ and MAPK Signaling Pathways. Inflammation, 2017. 40(1).

16. Manning, B. D. and A. Toker, AKT/PKB Signaling: Navigating the Network. Ce11, 2017. 169(3): p. 381-405.

17. Nüchel, J., et al., TGFB1 is secreted through an unconventional pathway dependent on the autophagic machinery and cytoskeletal regulators. Autophagy, 2018. 14(3): p. 465-486.

18. Piedra-Quintero, Z. L., et al., Myosin $1 F$ Regulates M1Polarization by Stimulating Intercellular Adhesion in Macrophages. Frontiers in Immunology, 2019. 9.

19. Vandenboom, R., Modulation of Skeletal Muscle Contraction by Myosin Phosphorylation. Compr Physiol, 2016. 7(1): p. 171-212.

20. Titus, M. A., Myosin-Driven Intracellular Transport. Cold Spring Harb Perspect Biol, 2018. 10 (3). 
602 21. Jung, G., X. Wu, and J.A. Hammer, 3rd, Dictyostelium mutants

603 lacking multiple classic myosin I isoforms reveal combinations

604 of shared and distinct functions. J Cell Biol, 1996. $133(2)$ : p.

$605 \quad 305-23$.

606 22. Jung, G. and J. A. Hammer, 3rd, Generation and characterization

607 of Dictyostelium cells deficient in a myosin I heavy chain

608 isoform. J Cell Biol, 1990. 110 (6) : p. 1955-64.

609 23. Wessels, D., et al., Myosin IB null mutants of Dictyostelium

610 exhibit abnormalities in motility. Cell Motil Cytoskeleton,

611 1991. 20(4): p. 301-15.

612 24. Temesvari, L. A., et al., Examination of the endosomal and

613 lysosomal pathways in Dictyostelium discoideum myosin I

614 mutants. J Cel1 Sci, 1996. 109 ( Pt 3) : p. 663-73.

615 25. Guo, Y., et al., An improved method for establishment of murine 616 retinal detachment model and its 3D vascular evaluation.

617 Experimental eye research, 2020. 193: p. 107949.

618 26. Gao, M., et al., xCT regulates redox homeostasis and promotes

619 photoreceptor survival after retinal detachment. Free Radic

620 Biol Med, 2020. 158: p. 32-43.

621 27. Henn, A., et al., The suitability of BV2 cells as alternative

622 model system for primary microglia cultures or for animal

623 experiments examining brain inflammation. ALTEX, 2009. 26(2):

624 p. $83-94$.

625 28. Zhou, T., et al., Microglia Polarization with M1/M2 Phenotype

626 Changes in rdl Mouse Model of Retinal Degeneration. Frontiers

627 in neuroanatomy, 2017. 11: p. 77.

628 29. Krigel, A., et al., Light-induced retinal damage using

629 different light sources, protocols and rat strains reveals LED

630 phototoxicity. Neuroscience, 2016. 339: p. 296-307.

631 30. Tisi, A., et al., Up-regulation of pro-angiogenic pathways and

632 induction of neovascularization by an acute retinal light

633 damage. Sci Rep, 2020. 10(1): p. 6376.

634 31. Salvermoser, M., et al., Myosin If is specifically required for 
635

636

637

638

639

640

641

642

643

644

645

646

647

648

649

650

651

652

653

654

655

656

657

658

659

660

661

662

663

664

665

666

667

neutrophil migration in $3 D$ environments during acute inflammation. Blood, 2018. 131(17): p. 1887-1898.

32. Wang, Y., et al., Myosinlf-mediated neutrophil migration contributes to acute neuroinflammation and brain injury after stroke in mice. Journal of neuroinflammation, 2019. 16(1): p. 77.

33. Tang, Y. and W. Le, Differential Roles of M1 and M2 Microglia in Neurodegenerative Diseases. Mol Neurobio1, 2016. 53(2): p. 1181-1194.

34. Hendrickx, D. A. E., et al., Staining of HLA-DR, Ibal and CD68 in human microglia reveals partially overlapping expression depending on cellular morphology and pathology. J Neuroimmunol, 2017. 309: p. 12-22.

35. Walker, D. G. and L.F. Lue, Immune phenotypes of microglia in human neurodegenerative disease: challenges to detecting microglial polarization in human brains. Alzheimers Res Ther, 2015. $7(1):$ p. 56.

36. Sosna, J., et al., Early long-term administration of the CSF1R inhibitor PLX3397 ablates microglia and reduces accumulation of intraneuronal amyloid, neuritic plaque deposition and prefibrillar oligomers in 5XFAD mouse model of Alzheimer's disease. Mol Neurodegener, 2018. 13(1): p. 11.

37. Tahmasebi, F., et al., Effect of the CSF1R inhibitor PLX3397 on remyelination of corpus callosum in a cuprizone-induced demyelination mouse model. J Cell Biochem, 2019. 120(6): p. 10576-10586.

38. Merry, T.L., et a1., The CSF1 receptor inhibitor pexidartinib (PLX3397) reduces tissue macrophage levels without affecting glucose homeostasis in mice. Int J Obes (Lond), 2020. 44(1): p. $245-253$.

39. Stansley, B., J. Post, and K. Hensley, A comparative review of cell culture systems for the study of microglial biology in Alzheimer's disease. J Neuroinflammation, 2012. 9: p. 115. 
668

669

670

671

672

673

674

675

676

677

678

679

680

681

682

683

684

685

686

687

688

689

690

691

692

693

694

695

696

697

698

699

700

40. Pardue, M. T. and R. S. Allen, Neuroprotective strategies for retinal disease. Progress in retinal and eye research, 2018. 65: p. $50-76$.

41. Daruich, A., et al., Iron is neurotoxic in retinal detachment and transferrin confers neuroprotection. Science advances, 2019. 5(1): p. eaau9940.

42. Chung, C. L., et al., Roles of Myosin-Mediated Membrane Trafficking in TGF- $\beta$ Signaling. Int J Mol Sci, 2019. 20(16).

43. McConne11, R. E. and M. J. Tyska, Leveraging the membrane cytoskeleton interface with myosin-1. Trends in cell biology, 2010. $20(7)$ : p. 418-426.

44. Heissler, S.M. and J.R. Sellers, Various Themes of Myosin Regulation. Journal of molecular biology, 2016. 428 (9 Pt B) : p. 1927-1946.

45. Piedra-Quintero, Z. L., et al., Myosin 1F Regulates M1Polarization by Stimulating Intercellular Adhesion in Macrophages. Frontiers in immunology, 2018. 9: p. 3118.

46. A, L., et al., Rescue of Retinal Degeneration in rd1 Mice by Intravitreally Injected Metformin. Frontiers in molecular neuroscience, 2019. 12: p. 102.

47. Mukherjee, S., et al., A Microglial Signature Directing Human Aging and Neurodegeneration-Related Gene Networks. Front Neurosci, 2019. 13: p. 2.

48. Silverman, S.M. and W. T. Wong, Microglia in the Retina: Roles in Development, Maturity, and Disease. Annual review of vision science, 2018. 4: p. 45-77.

49. Du, L., et a1., Role of Microglia in Neurological Disorders and Their Potentials as a Therapeutic Target. Molecular neurobiology, 2017. 54(10): p. 7567-7584.

50. Block, M. L., L. Zecca, and J. -S. Hong, Microglia-mediated neurotoxicity: uncovering the molecular mechanisms. Nature reviews. Neuroscience, 2007. 8(1): p. 57-69.

51. Colonna, M. and 0. Butovsky, Microglia Function in the Central 
Nervous System During Health and Neurodegeneration. Annual review of immunology, 2017. 35: p. 441-468.

52. Okunuki, Y., et al., Microglia inhibit photoreceptor cell death and regulate immune cell infiltration in response to retinal detachment. Proceedings of the National Academy of Sciences of the United States of America, 2018. 115(27): p. E6264-E6273.

53. Sun, Y., et al., Signaling pathway of MAPK/ERK in cell proliferation, differentiation, migration, senescence and apoptosis. Journal of receptor and signal transduction research, 2015. 35(6): p. 600-604.

54. Kyriakis, J.M. and J. Avruch, Mammalian MAPK signal transduction pathways activated by stress and inflammation: a 10-year update. Physiological reviews, 2012. 92(2): p. 689-737.

55. Santa-Cecília, F. V., et al., Doxycycline Suppresses Microglial Activation by Inhibiting the p38 MAPK and NF-kB Signaling Pathways. Neurotoxicity research, 2016. $29(4)$ : p. 447-459.

56. Feng, N., Y. Jia, and X. Huang, Exosomes from adipose-derived stem cells alleviate neural injury caused by microglia activation via suppressing $N F-k B$ and MAPK pathway. Journal of neuroimmunology, 2019. 334: p. 576996.

57. Nam, H. Y., et a1., Ibrutinib suppresses LPS-induced neuroinflammatory responses in BV2 microglial cells and wildtype mice. J Neuroinflammation, 2018. 15(1): p. 271.

58. Zhang, Y., et al., Kinase AKT controls innate immune cell development and function. Immunology, 2013. $140(2)$ : p. 143-52.

59. Qin, S., et al., Sulforaphane attenuates microglia-mediated neuronal necroptosis through down-regulation of $M A P K / N F-\kappa B$ signaling pathways in LPS-activated BV-2 microglia. Pharmacological research, 2018. 133: p. 218-235.

60. Frakes, A.E., et al., Microglia induce motor neuron death via the classical $N F-\kappa B$ pathway in amyotrophic lateral sclerosis. Neuron, 2014. 81(5): p. 1009-1023.

61. Rafiq, N.B.M., et al., A mechano-signalling network linking 
734

735

736

737

738

739

740

741

742

743

744

745

746

747

748 microtubules, myosin IIA filaments and integrin-based adhesions. Nature Materials, 2019. 18(6): p. 638-649.

62. Choi, C., et al., Integrin $\beta 1$, myosin light chain kinase and myosin IIA are required for activation of PI3K-AKT signaling following MEK inhibition in metastatic triple negative breast cancer. Oncotarget, 2016. $7(39)$ : p. 63466-63487.

63. Chinthalapudi, K., et al., Mechanism and specificity of pentachloropseudilin-mediated inhibition of myosin motor activity. J Biol Chem, 2011. 286(34): p. 29700-8.

64. Heissler, S.M. and J.R. Sellers, Various Themes of Myosin Regulation. J Mol Biol, 2016. 428 (9 Pt B) : p. 1927-46.

65. Rauscher, A., et al., Targeting Myosin by Blebbistatin Derivatives: Optimization and Pharmacological Potential. Trends Biochem Sci, 2018. $43(9)$ : p. 700-713. 
Figure legends

Figure 1

Transcriptome analysis of human retinal detachment. A. Identification of differential expressed genes (DRGs) were set as $|F C|>1.5$ and $p<0.05$. Red dots on the volcano plot $(A)$ represent up-regulated genes while blue dots represent down-regulated genes. B-C. Gene ontology analysis. Cellular components analysis (B) and biological process analysis (C) showed possible functions of DEGs. D. Gene-set enrichment analysis (GSEA) also revealed possible pathways possibly correlated to RD, including inflammatory response. E-F. Expression pattern of myosins. Heatmap (E) demonstrated the most evaluated myosin, myosin 1f, with a FC=1.786 (F). G. The fold changes of at different RD duration.

\section{Figure 2}

Myosin $1 \mathrm{f}$ is up-regulated after mouse model of retinal detachment. A. The expression of myosin $1 \mathrm{f}$ at day 1 , day 3 , day 7 , it reached to peak at day $3(\mathrm{~A})$. 
The expression value is calculated by the optical density ration of myosin $1 f$ and GAPDH. B. Representative image of eye sections stained for F4/80 (green), myosin $1 \mathrm{f}$ (red) and dapi (blue). The eyeballs were taken down at day 3 after RD. C. Representative image of eye sections stained for IBA1 (green), myosin 1f (red) and dapi (blue) (day 3). D. Eye sections stained for GFAP (green), myosin $1 f$ (red) and dapi (blue) (day 3). E-F. Myosin $1 \mathrm{f}$ is also up-regulated in rd1 mouse(E) (day 7 after birth) and light-injured retina(F) (day 5). G. Retinal sections of rd1 mice (day 7 after birth) stained for F4/80 (green), myosin $1 \mathrm{f}$ (red) and dapi (blue). H. Retinal sections of light-injured retina (day 5) stained for F4/80 (green), myosin $1 f$ (red) and dapi (blue).

Figure 3

Observation of myosin 1 f $/-$ mice after retinal detachment model. A. Two groups of mice, myosin 1f-I- and WT, were sacrificed at day 3. B-C. HE staining showed the thickness of ONL on detached retina (day 3) of two groups (B). Scale bar, $100 \mu \mathrm{m}$. Measurements of ONL were taken by image $\mathrm{J}(\mathrm{C})$, and data 
were presented as mean \pm SEM, unpaired $t$ test, ${ }^{*} \mathrm{P}<0.05,{ }^{* *} \mathrm{P}<0.01$, ${ }^{* * *} \mathrm{P}<0.001,{ }^{* * * * P}<0.0001$. D-E. Representative tunel staining in ONL (in green) (3 days after induction of RD) showed apoptosis of photoreceptors, scale bar, $100 \mu \mathrm{m}(\mathrm{D})$, quantification of tunel positive cells in ONL (E) revealed the significance, data were presented as mean $\pm S E M$, unpaired t test, ${ }^{*} \mathrm{P}<0.05$, ${ }^{* *} \mathrm{P}<0.01,{ }^{* * *} \mathrm{P}<0.001,{ }^{* * *} \mathrm{P}<0.0001$

Figure 4

Knock out of myosin $1 f$ affects the function of microglia. A. Flatmount of detached retina of WT and myosin 1f -/- mice, stained by IBA1 (in red), showed morphology of microglia. Scale bar, $25 \mu \mathrm{m}$. B-C. Skeleton analysis (B) and sholl analysis (C) were conducted to quantify microglia morphology. The less average branch length is, the more activated microglia is; Similarly, the less interaction number is, the more activated microglia is. D-E. ELISA analysis of IL-1 $(\mathrm{D})$ and TNF- $\alpha(E)$ in detached retina of WT and myo1f KO mice (day 3). F-G. Qpcr analysis of IL-1 $\beta$ and TNF- $\alpha$ in detached retina at day $3(F)$ and 
CD68 (G) at day 1. Data were presented as mean \pm SEM, unpaired $t$ test, ${ }^{*} \mathrm{P}<0.05,{ }^{* *} \mathrm{P}<0.01,{ }^{* * *} \mathrm{P}<0.001,{ }^{* * * *} \mathrm{P}<0.0001$

Figure 5

Elimination of microglia reverse the protective effect of myosin $1 \mathrm{f}$ deficiency. A. PLX3397 was given to WT and myosin 1f-/- mice everyday from day 1 to day 6 , the mice were sacrificed at day 7,3 days after mouse model of retinal detachment (day 4). B. Representative HE staining showed the thickness of ONL on detached retina (scale bar, $50 \mu \mathrm{m}$ ), representative tunel staining in ONL (in green) ( 3 days after induction of RD) (scale bar, 100 $\mu \mathrm{m}$ ) showed apoptosis of photoreceptors after microglia elimination. C. Quantification of ONL thickness and tunel positive cells in ONL between two groups. D-E. Qpcr analysis demonstrated the fold change of IL-1 $\beta$, TNF- $\alpha$ and CD68 (day 3), data were presented as mean $\pm S E M$, unpaired $t$ test, ${ }^{*} P<0.05,{ }^{* *} P<0.01$, ${ }^{* * *} \mathrm{P}<0.001,{ }^{* * * *} \mathrm{P}<0.0001$ 
Figure 6

Myosin $1 f$ affects the activation of microglia by regulating NF-kb and MAPK pathways. A. In vitro, western blot showed that myosin $1 f$ is up-regulated after stimulated by LPS (100ng/ml) for 24 hours, the expression value is calculated by the optical density ration of myosin $1 f$ and GAPDH. B. The expression of IL$1 \beta$ (qpcr) and TNF- $\alpha$ (ELISA) at every time point. C-D. After siRNA on BV2 cell lines, we detected the expression of myosin $1 f$ by western blot (C). Three sequences of siRNA $(768,1413,2772)$ were designed to knock down myosin 1f, 2662 were chosen to further confirm the efficiency (D). E. ELISA analysis of IL$1 \beta$ and TNF- $\alpha$ after knock down myosin 1f. F. In vitro, wb analysis showed expression pattern of NF-kb and MAPK pathways. All the quantification of phosphorylation protein is calculated by the optical density ration of phosphorylation protein and its corresponding protein. G. All the quantification of phosphorylation protein is calculated by the optical density ration of phosphorylation protein and GAPDH. Data were presented as mean \pm SEM, unpaired t test, ${ }^{*} \mathrm{P}<0.05,{ }^{* *} \mathrm{P}<0.01,{ }^{* * *} \mathrm{P}<0.001,{ }^{* * * * \mathrm{P}}<0.0001$. 
Supplementary figure 1

A. Gene-set enrichment analysis (GSEA) also revealed possible pathways possibly correlated to RD, including interferon-gamma response, IL-6-STAT3 signaling, complement, IL-2-STAT5 signaling.

Supplementary figure 2

Myosin $1 f$ deficiency does not affect the structure and function of retina. A-D.

OCT $(A, B)$ and HE (C, D) staining reflects the thickness of WT and myosin 1fI- mouse retina. Data were presented as mean $\pm S E M$, unpaired $t$ test, n.c.

$P>0.05$. E. Electroretinogram of $W T$ and myosin $1 f$ mice, more than 20 weeks- year-old.

Data were presented as mean $\pm S E M$, unpaired $t$ test, ${ }^{*} P<0.05,{ }^{* *} P<0.01$, ${ }^{* * *} \mathrm{P}<0.001,{ }^{* * * *} \mathrm{P}<0.0001$ 
A-B. Fundus photography (A) and HE (B) showed detachment of RPE and photoreceptor (day 3). C. IBA1+ cell counts of the stretched preparation of retina after retinal detachment. D The cell count of both F4/80+ and IBA1+ cells infiltrated into outer nuclear layer. Data were presented as mean $\pm S E M$, unpaired $\mathrm{t}$ test, ${ }^{*} \mathrm{P}<0.05,{ }^{* *} \mathrm{P}<0.01,{ }^{* * *} \mathrm{P}<0.001,{ }^{* * * *} \mathrm{P}<0.0001$. 


\section{Figures}

A

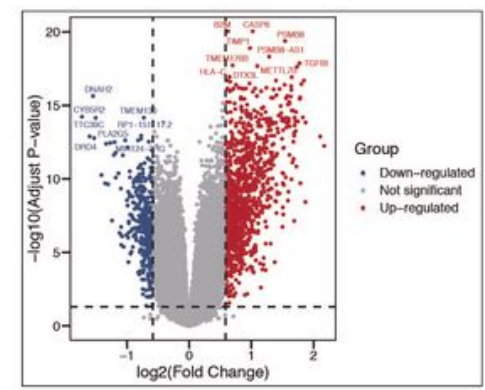

D

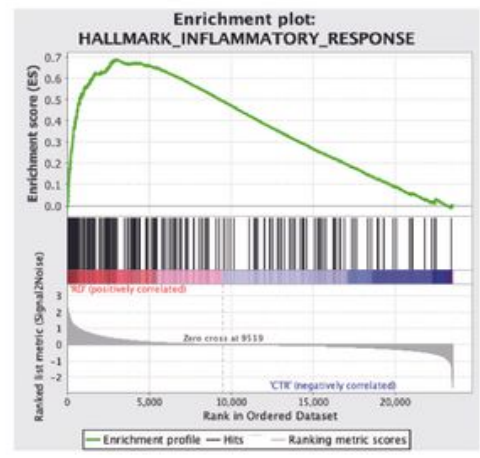

$\mathrm{F}$

\begin{tabular}{|c|c|}
\hline Gene name & Fold Change \\
\hline MYO1F & 1.78609924 \\
\hline MYO3A & 1.5336209 \\
\hline MYO5C & 1.47770792 \\
\hline
\end{tabular}

B

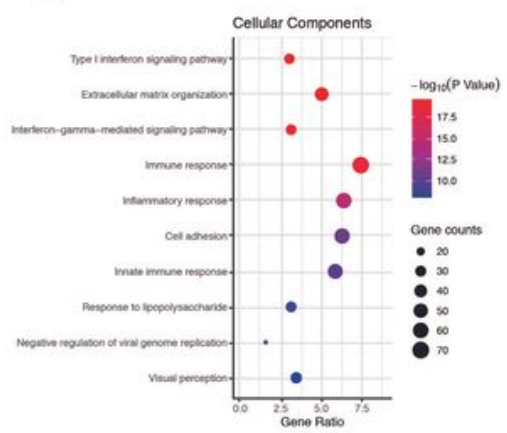

C

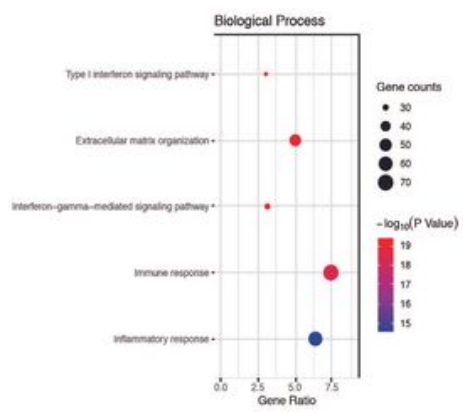

$E$

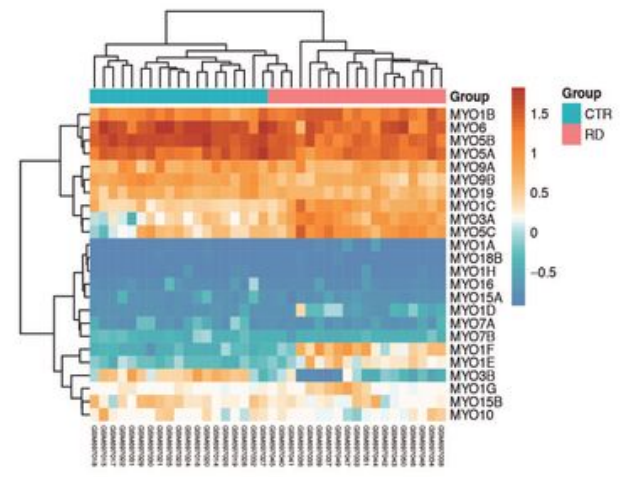

G

\begin{tabular}{|c|c|}
\hline Duration & Fold Change \\
\hline within one month & 2.19493181 \\
\hline 1 month - 3 month & 1.70387994 \\
\hline more than 3 month & 1.76519891 \\
\hline
\end{tabular}

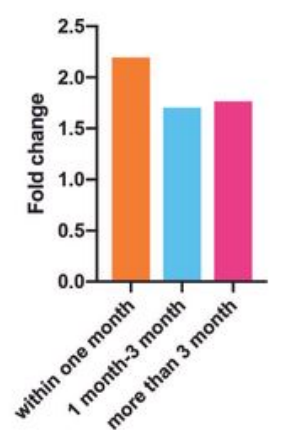

\section{Figure 1}

Transcriptome analysis of human retinal detachment. A. Identification of differential expressed genes (DRGs) were set as $|F C|>1.5$ and $p<0.05$. Red dots on the volcano plot (A) represent up-regulated genes while blue dots represent down-regulated genes. B-C. Gene ontology analysis. Cellular components 
analysis (B) and biological process analysis (C) showed possible functions of DEGs. D. Gene-set enrichment analysis (GSEA) also revealed possible pathways possibly correlated to RD, including inflammatory response. E-F. Expression pattern of myosins. Heatmap (E) demonstrated the most evaluated myosin, myosin 1f, with a $F C=1.786(F)$. G. The fold changes of at different RD duration.

A

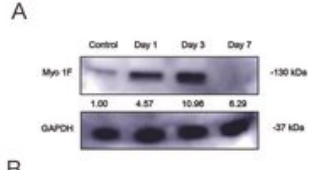

B

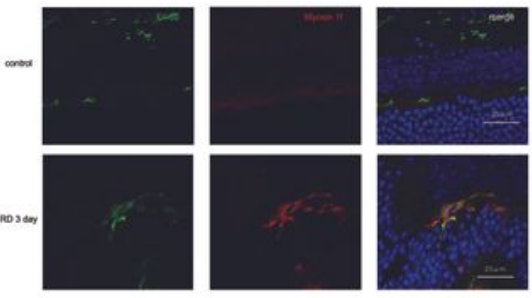

C

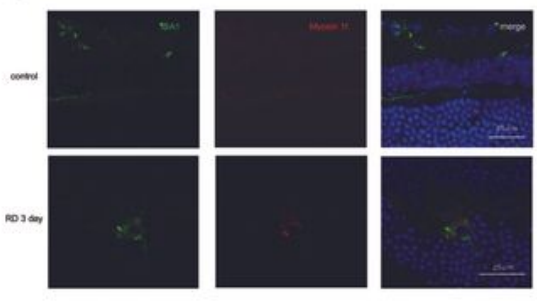

D
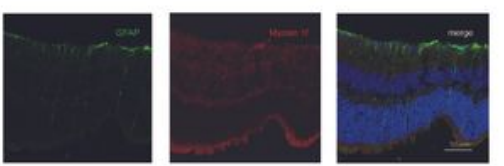

$E$

$\mathrm{F}$

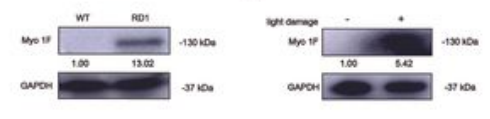

G
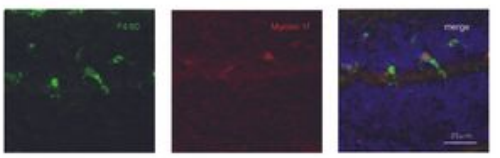

$\mathrm{H}$
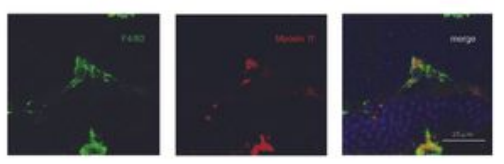

Figure 2 
Myosin $1 \mathrm{f}$ is up-regulated after mouse model of retinal detachment. A. The expression of myosin $1 \mathrm{f}$ at day 1 , day 3 , day 7 , it reached to peak at day $3(A)$. The expression value is calculated by the optical density ration of myosin $1 \mathrm{f}$ and GAPDH. B. Representative image of eye sections stained for F4/80 (green), myosin $1 \mathrm{f}$ (red) and dapi (blue). The eyeballs were taken down at day 3 after RD. C.

Representative image of eye sections stained for IBA1 (green), myosin $1 \mathrm{f}$ (red) and dapi (blue) (day 3). D. Eye sections stained for GFAP (green), myosin $1 f$ (red) and dapi (blue) (day 3). E-F. Myosin $1 \mathrm{f}$ is also upregulated in rd1 mouse(E) (day 7 after birth) and light-injured retina(F) (day 5). G. Retinal sections of rd1 mice (day 7 after birth) stained for F4/80 (green), myosin $1 f$ (red) and dapi (blue). H. Retinal sections of light-injured retina (day 5) stained for F4/80 (green), myosin $1 f$ (red) and dapi (blue). 


\section{A}
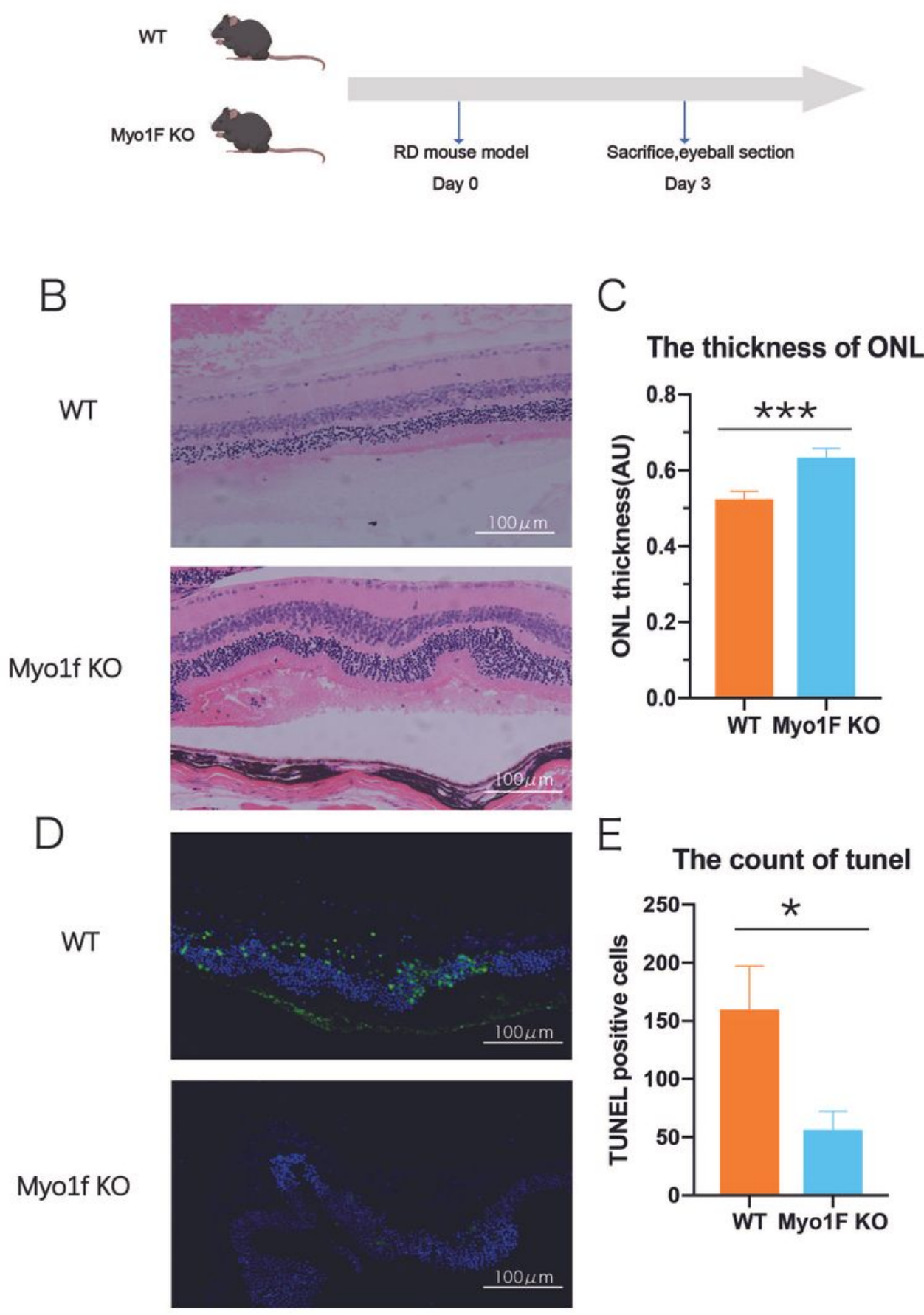

\section{Figure 3}

Observation of myosin $1 \mathrm{f}-/-$ mice after retinal detachment model. A. Two groups of mice, myosin $1 \mathrm{f}-/-$ and WT, were sacrificed at day 3. B-C. HE staining showed the thickness of ONL on detached retina (day 3 ) of two groups (B). Scale bar, $100 \mu \mathrm{m}$. Measurements of ONL were taken by image J (C), and data were

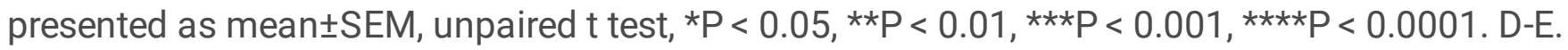
Representative tunel staining in ONL (in green) (3 days after induction of RD) showed apoptosis of 
photoreceptors, scale bar, $100 \mu \mathrm{m}(\mathrm{D})$, quantification of tunel positive cells in ONL (E) revealed the significance, data were presented as mean $\pm S E M$, unpaired $t$ test, ${ }^{*} P<0.05,{ }^{\star \star} P<0.01, \star \star \star * P<0.001$, ${ }^{\star \star \star \star} P$ $<0.0001$.

A
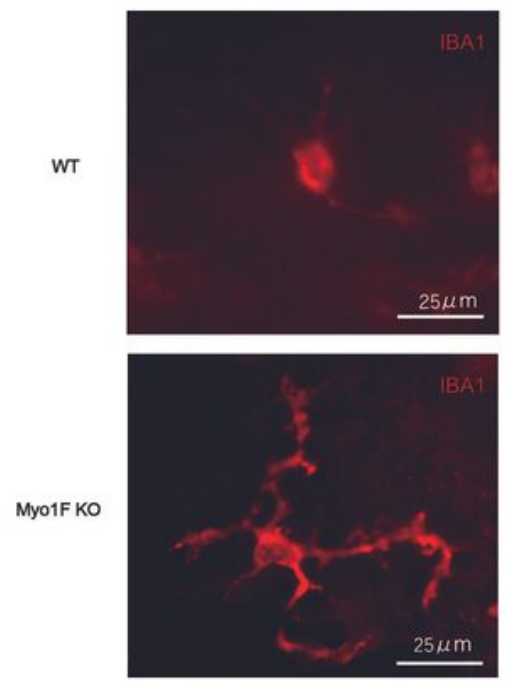

$\mathrm{D}$

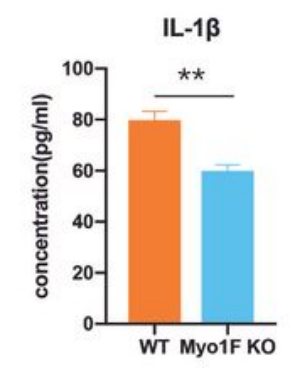

$\mathrm{F}$

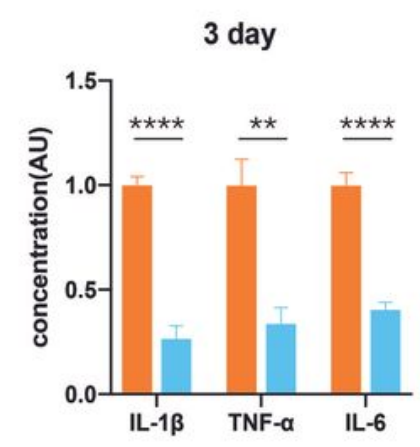

B
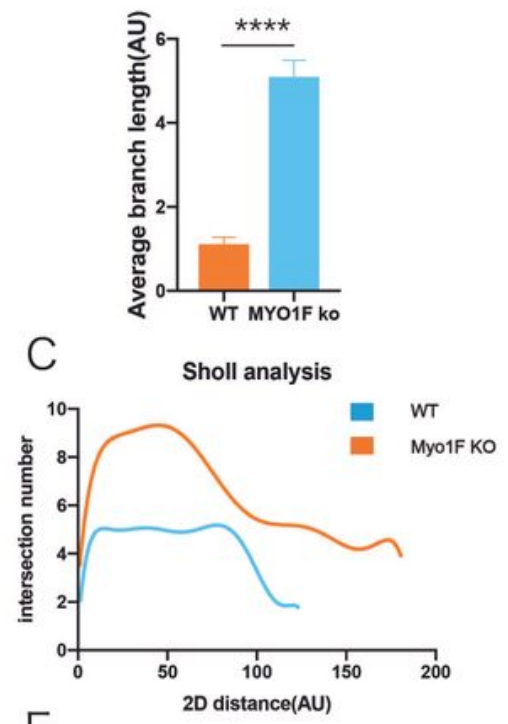

E

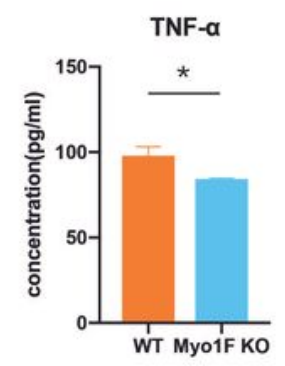

G

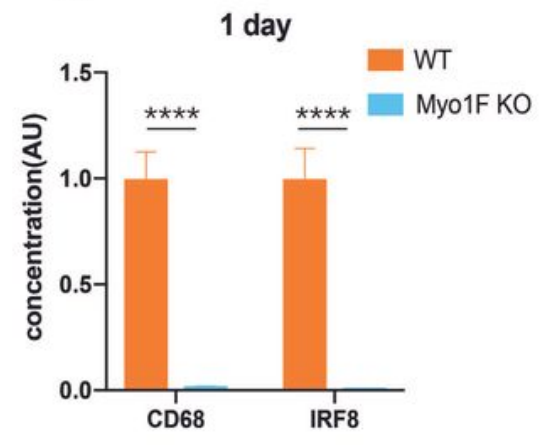

\section{Figure 4}

Knock out of myosin $1 \mathrm{f}$ affects the function of microglia. A. Flatmount of detached retina of WT and myosin $1 \mathrm{f}$-/- mice, stained by IBA1 (in red), showed morphology of microglia. Scale bar, $25 \mu \mathrm{m}$. B-C. 
Skeleton analysis (B) and sholl analysis (C) were conducted to quantify microglia morphology. The less average branch length is, the more activated microglia is; Similarly, the less interaction number is, the more activated microglia is. D-E. ELISA analysis of IL-1 1 (D) and TNF- $\alpha(E)$ in detached retina of WT and myo1f KO mice (day 3). F-G. Qpcr analysis of IL-1 $\beta$ and TNF- $\alpha$ in detached retina at day 3 (F) and CD68

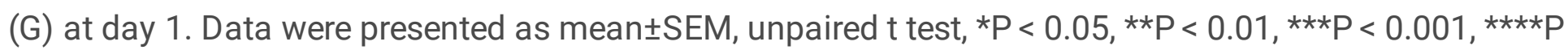
$<0.0001$.

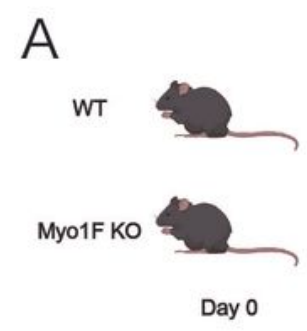

$\mathrm{B}$

WT
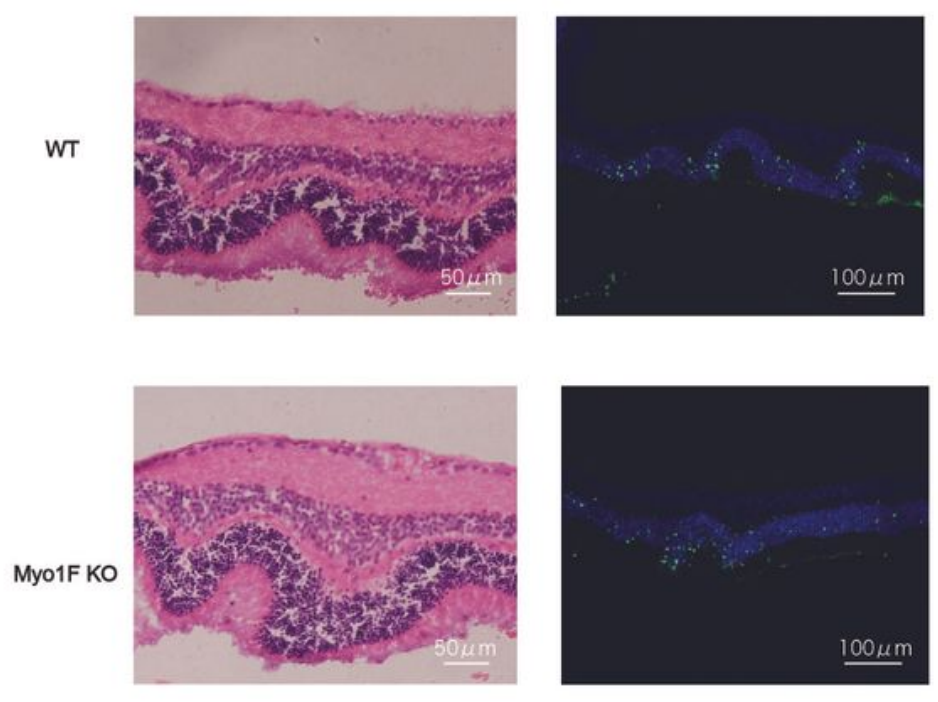

D

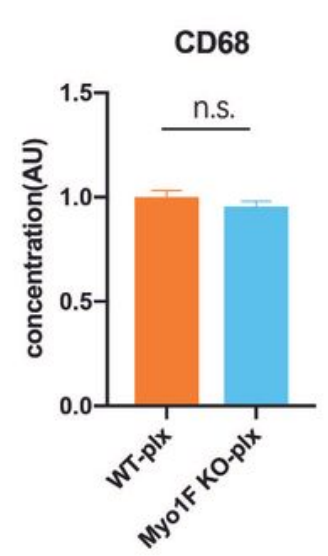

E

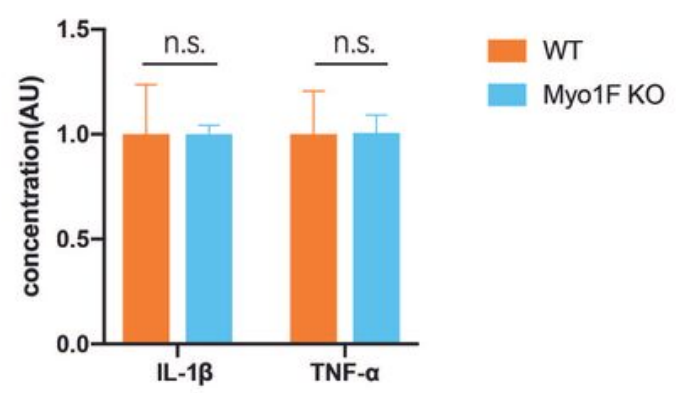

\section{E}
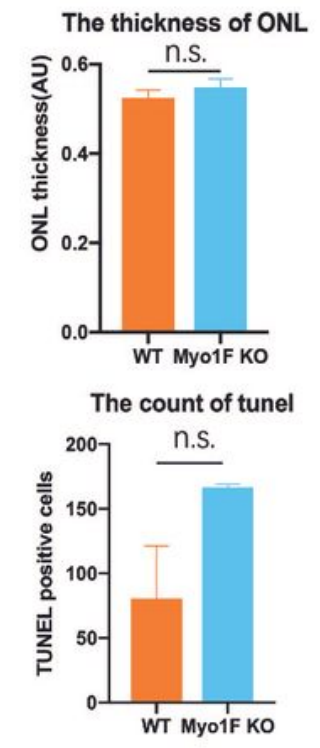

Sacrifice,eyeball section Day 7

C 
Elimination of microglia reverse the protective effect of myosin $1 \mathrm{f}$ deficiency. A. PLX3397 was given to WT and myosin $1 \mathrm{f}-/$ - mice everyday from day 1 to day 6 , the mice were sacrificed at day 7, 3 days after mouse model of retinal detachment (day 4). B. Representative HE staining showed the thickness of ONL

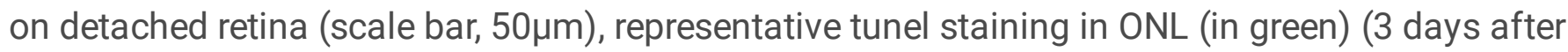
induction of RD) (scale bar, $100 \mu \mathrm{m}$ ) showed apoptosis of photoreceptors after microglia elimination. C. Quantification of ONL thickness and tunel positive cells in ONL between two groups. D-E. Qpcr analysis demonstrated the fold change of IL-1 $\beta, T N F-\alpha$ and CD 68 (day 3), data were presented as mean \pm SEM,

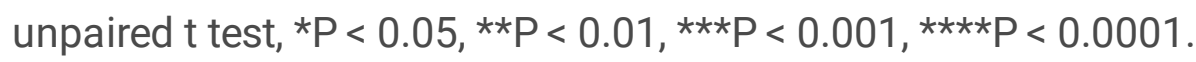



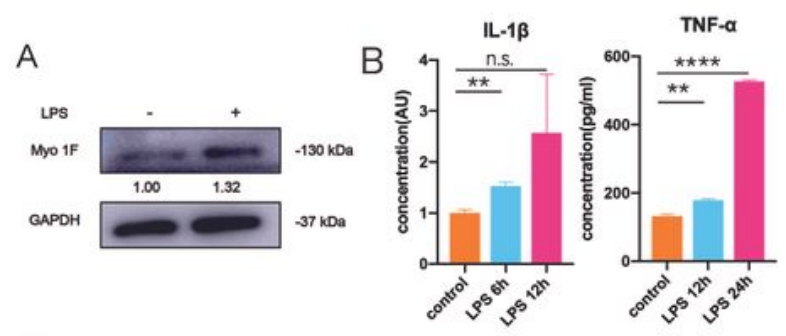

C

D
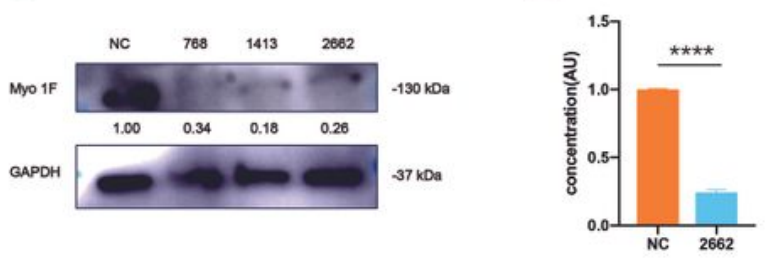

E
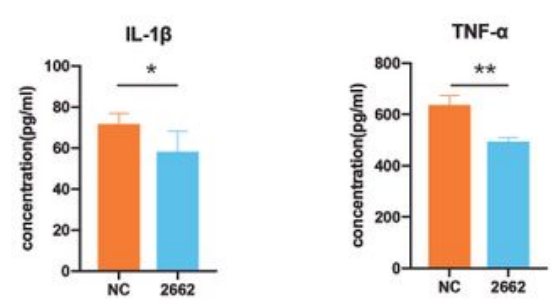

F

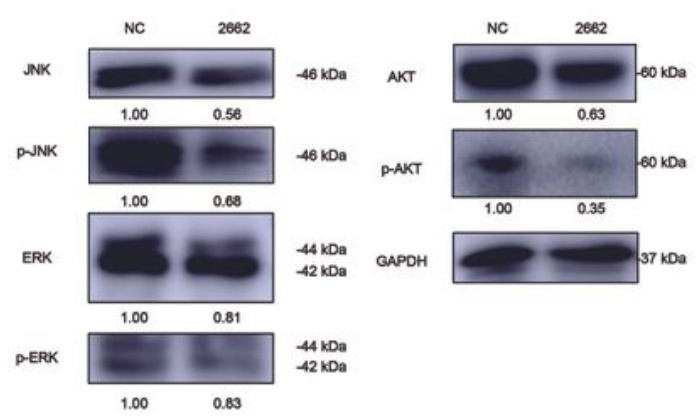

G

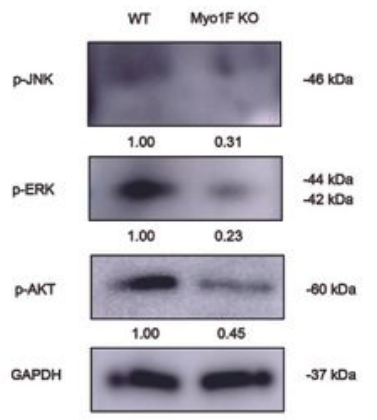

\section{Figure 6}

Myosin $1 \mathrm{f}$ affects the activation of microglia by regulating NF-kb and MAPK pathways. A. In vitro, western blot showed that myosin $1 \mathrm{f}$ is up-regulated after stimulated by LPS (100ng/ml) for 24 hours, the expression value is calculated by the optical density ration of myosin $1 \mathrm{f}$ and GAPDH. B. The expression of IL-1 $\beta$ (qpcr) and TNF-a (ELISA) at every time point. C-D. After siRNA on BV2 cell lines, we detected the expression of myosin $1 \mathrm{f}$ by western blot $(\mathrm{C})$. Three sequences of siRNA $(768,1413,2772)$ were designed to 
knock down myosin 1f, 2662 were chosen to further confirm the efficiency (D). E. ELISA analysis of IL-1 $\beta$ and TNF-a after knock down myosin 1f. F. In vitro, wb analysis showed expression pattern of NF-kb and MAPK pathways. All the quantification of phosphorylation protein is calculated by the optical density ration of phosphorylation protein and its corresponding protein. G. All the quantification of phosphorylation protein is calculated by the optical density ration of phosphorylation protein and

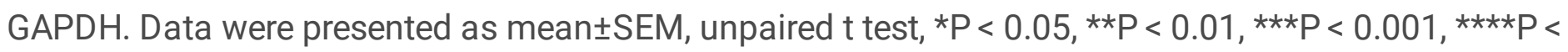
0.0001 .

\section{Supplementary Files}

This is a list of supplementary files associated with this preprint. Click to download.

- supplementary1.jpg

- supplementary2.jpg

- supplementary3.jpg 